Pacific Journal of Mathematic 


\title{
GEOMETRY AND THE RADON-NIKODYM THEOREM IN STRICT MACKEY CONVERGENCE SPACES
}

\section{DAVID Gilliam}

\begin{abstract}
The main purpose of this paper is to indicate a technique for extending certain types of results which are known for Banach spaces to the corresponding results in more general locally convex topological vector spaces. We shall extend these results to the class of locally convex spaces possessing the strict Mackey convergence [SMC] property. The technique involves a natural embedding into a Banach space and an application of the Banach space result. Loosely speaking, we have that those properties of closed bounded sets in Banach spaces that do not depend upon any open set will yield analogous results for closed bounded sets in quasicomplete locally convex spaces with the SMC property.
\end{abstract}

Peck [16] and Saab [20] have used a constructive embedding technique for extending results to Fréchet spaces and we shall see that for spaces with the SMC property the embedding used is obtained in a very natural manner without necessity of construction.

In the first section we shall be concerned with vector integration, the Radon-Nikodym Theorem [RNT], and the Radon-Nikodym Property [RNP] for spaces with the SMC property. Rieffel $[18,19]$, Maynard [13], and others [1,5] have proved a RNT for the Bochner and the Dunford second integral in Banach spaces. By modifying their techniques, Chi [3] has extended this theorem to Fréchet spaces. We shall extend the Dunford second integral to spaces with the SMC property and obtain the RNT directly from the Banach space result.

Banach spaces with the RNP have been characterized $[5,9,10$, 13,17 ] in terms of the geometric and extremal structure of their closed bounded convex sets. Saab [20] and Chi [3] have extended some of these results to Fréchet spaces for a Bochner type integral. We shall obtain these results for our integration theory in spaces with the SMC property.

The second section deals with the separation of closed bounded convex sets, denseness of support points for closed bounded convex sets and denseness of support functionals for closed bounded convex sets. We shall give two results on weakly compact subsets of spaces with the SMC property. Bishop and Phelps [1] gave several results on support points of convex sets in Banach spaces and Peck [16] has given analogs of some of them in Fréchet spaces. We 
shall show that many of the Bishop and Phelps results extend naturally to spaces with the SMC property. Finally, in [4] it was shown that a weakly compact subset of a Banach space is affinely homeomorphic to a subset of some reflexive Banach space and that a weakly compact operator from a locally convex space to a Banach space factors through a reflexive Banach space. In the same paper it was indicated how these results extend to Fréchet spaces. We demonstrate that these results hold for spaces with the SMC property, the results following directly from the Banach space results.

1. Definitions and notations. For a locally convex topological vector space $E$ over the reals, $\boldsymbol{R}$, we denote by $\{\rho\}$ a fundamental family of continuous seminorms which determine the topology of $E$, by $E^{*}$ the algebraic dual of $E$ and by $E^{\prime}$ the continuous dual of $E$. If $A$ is a bounded disked set, then we shall denote by $E_{A}$ the normed linear space consisting of the linear span of $A$ in $E$ with the norm $\|x\|_{A}=\inf \{\lambda: \lambda>0, x \in \lambda A\}$. We shall say that $E$ has the strict Mackey convergence property [SMC] if for every bounded disked set $A$ in $E$ there is a bounded disked set $B$ containing $A$ such that the topologies of $E$ and $E_{B}$ agree on $A$. We observe that the uniform structures on $A$ induced by $E$ and $E_{B}$ also coincide [7, page 75 ]. If $E$ is also quasicomplete (every closed bounded set is complete), then $E_{B}$ is a Banach space. We shall say that a space with the SMC property is a SMC space, and a quasicomplete space with the SMC property a QSMC space.

The SMC property was introduced by Grothendieck [6], who showed that metrizable spaces are SMC spaces. We say a locally convex space $E$ satisfies the Mackey convergence [MC] condition if for every sequence $\left\{x_{n}\right\}$ converging to zero in $E$, there is a bounded disked set $B$ in $E$ containing $\left\{x_{n}\right\}$ such that $\left\{x_{n}\right\}$ converges to zero in $E_{B}$. Grothendieck showed that a locally convex space with a fundamental sequence of bounded sets (a sequence of bounded sets in $E$ such that every bounded set in $E$ is contained in a member of the sequence) has the SMC property if and only if it has the MC property and the topology of $E$ is metrizable on bounded sets. This gives that strict inductive limits of Banach spaces and quasicomplete $(L F)$-space which have a fundamental sequence of bounded sets, are QSMC spaces. We also note that the countable product of spaces with SMC property has the SMC property, subspaces of spaces with the SMC property have the SMC property and any topological direct sum of a family of spaces with the SMC property has the SMC property.

Throughout, we denote by $(T, \Sigma, \mu)$ a positive finite measure 
space over $\Sigma$, a sigma algebra of subsets of $T$ and let $\Sigma^{+}=\{S \in \Sigma$ : $\mu(S)>0\}$. A set property $P$ is said to be local in $\Sigma^{+}$if for every $S$ in $\Sigma^{+}$, there is a $S_{1} \subset S, S_{1}$ in $\Sigma^{+}$such that $S_{1}$ has property $P$.

Definition 1. For a set $K \subset E$, we define $d(K)=$ closure $\left\{\sum_{i \in I} \alpha_{i} x_{i}:\left\{x_{i}\right\} \subset K, \Sigma\left|\alpha_{i}\right| \leqq 1\right.$, I finite $\}$. We call $d(K)$ the closed disked hull of $K$.

Definition 2. If $m: \Sigma \rightarrow E$ is a countably additive vector measure, then the average range of $m$ on $S$ in $\Sigma^{+}$is $A_{S}(m)=$ $\left\{m\left(S^{\prime}\right) / \mu\left(S^{\prime}\right): S^{\prime} \subset S, S^{\prime} \in \Sigma^{+}\right\}$.

DEFINITION 3. If $m: \Sigma \rightarrow E$ is a countably additive vector measure, then $m$ has locally small average range if for every $\rho \in\{\rho\}, \varepsilon>0$ and $S \in \Sigma^{+}$, there is an $S_{1} \subset S, S_{1} \in \Sigma^{+}$such that $\rho$-diam $\left(A_{S_{1}}(m)\right)$ is less than $\varepsilon$, i.e.; $\rho(x-y)<\varepsilon, \forall x, y \in A_{S_{1}}(m)$.

Definition 4. A set $K$ in $E$ is dentable if and only if for every $\rho \in\{\rho\}$ and $\varepsilon>0$, there is an $x \in K$ such that $x \notin \bar{c}\left(K \backslash S_{\rho}^{\varepsilon}(x)\right)$. Here $\bar{c}(\cdot)$ denotes the closed convex hull and $S_{\varepsilon}^{\rho}(x)=\{y \in E: \rho(x-y)<\varepsilon\}$.

DefINITION 5. Suppose $E$ is a QSMC space. A function $f: T \rightarrow E$ is integrable if and only if (i) there is a sequence of simple functions $\left\{f_{n}\right\}$ which converge to $f$ almost uniformly on $T$; (ii) $\left\{\int_{S} f_{n} d \mu\right\}_{n}$ converges for each $S \in \Sigma$. Then we define $\int_{S} f d \mu=$ $\lim _{n} \int_{S} f_{n} d \mu$, for every $S \in \Sigma$. Note that for Banach spaces this is the Dunford second integral or equivalently the Pettis integral for strongly measurable functions.

We note that many of the following results are ture for more general locally convex spaces with a slight modification of the above definition of integrable functions, but to preserve the unity and purpose of the paper we only indicate the results for QSMC spaces.

THEOREM. [RNT] Suppose $E$ is a QSMC space and $(T, \Sigma, \mu)$ is a positive finite measure space. If $m: \Sigma \rightarrow E$ is a countably additive vector measure, $m \ll \mu$, and $m$ has locally bounded average range, then the following are equivalent:

(1) There is an integrable function $f: T \rightarrow E$ such that $m(S)=$ $\int_{S} f d \mu, \forall S \in \Sigma$.

(2) $m$ has locally relatively compact average range.

(3) $m$ has locally relatively weakly compact average range.

(4) $m$ has locally dentable average range. 
(5) $m$ has locally small average range.

Proof. We show (1) $\Leftrightarrow(2) \Rightarrow(3) \Rightarrow(4) \Rightarrow(5) \Rightarrow(2)$ and where the proof is only a slight modification of the Banach space case the proof is omitted.

$(1) \Rightarrow(2)$ : The proof is exactly that given by Rieffel [19, Page 472] using an essential range argument.

$(2) \Rightarrow(1)$ : Using the exhaustion principle of Maynard [14, Page 450] we decompose $T$ into a countable disjoint collection of sets $\left\{T_{i}\right\} \subset \Sigma^{+}$such that $\forall i, A_{T_{i}}(m)$ is relatively compact. Let $D_{n}=$ $\mu\left(T_{n}\right) \cdot d\left(A_{T_{n}}(m)\right)$ where $d\left(A_{T_{n}}(m)\right)$ is the closed disked hull of $A_{T_{n}}(m)$. Hence the range of $\left.m_{n} \equiv m\right|_{T_{n}}: T_{n} \cap \Sigma \rightarrow E$ is contained in $D_{n}$. Now by the QSMC property there is a collection of subsets of $E,\left\{\hat{D}_{n}\right\}$, $D_{n} \subset \hat{D}_{n}$, which are closed bounded and disked and such that the topologies of $E$ and $E_{\widehat{D}_{n}}$ agree on $D_{n}$. Thus $m_{n}$ is a countable additive vector measure into $E_{\widehat{D}_{n}}$ with relatively compact average range and hence has a Bochner integrable density, $f_{n}: T_{n} \rightarrow E_{\widehat{D}_{n}}$. By a standard piecing argument [14, Page 455; 3, Page 14] we obtain a density for $m$.

$(2) \Rightarrow(3)$ : If $K \subset E$ is relatively compact, then $K$ is relatively weakly compact.

$(3) \Rightarrow(4)$ : Claim: If $K \subset E$ is relatively weakly compact, then $K$ is dentable.

Proof of the claim: If $K \subset E$ is relatively weakly compact, then by Krein's theorem, we have $d(K)$ is weakly compact. Take $\widehat{d(K)} \supset d(K)$ by the QSMC property so that the topologies of $E$ and $E_{d(K)}$ agree on $d(K)$; then using an argument of Grothendeick [7, Page 61 ] we have that $K$ is relatively weakly compact in $E_{d(K)}$. This implies that $K$ is dentable in $E_{d(\widehat{K})}$ and because the topologies and uniform structures agree on $d(K)$, it is easily checked that $K$ is dentable in $E$.

$(4) \Rightarrow(5)$ : Take $S \in \Sigma^{+}, \rho \in\{\rho\}$ and $\varepsilon>0$, then by (4) there is an $S_{1} \subset S, S_{1} \in \Sigma^{+}$, such that $A_{S_{1}}(m)$ is dentable and bounded in $E$. Define $K=\mu\left(S_{1}\right) \cdot d\left(A_{S_{1}}(m)\right)$ and take $\hat{K} \supset K$ by the QSMC property as above; then $\left.m_{1} \equiv m\right|_{S_{1}}: \Sigma \cap S_{1} \rightarrow E_{\widehat{K}}$ is a countably additive vector measure with range contained in $K$. Now $m_{1}$ has locally dentable average range in $E_{\widehat{K}}$ and hence it has locally small average range in $E_{\widehat{K}}$ by [14]. By our selection of $\hat{K}$ we have that $\left.m\right|_{S_{1}}$ has locally small average range in $E$.

$(5) \Longrightarrow(2)$ : This follows just as in $(4) \Rightarrow(5)$.

DEFINITION 6. If $E$ is a QSMC space, then $E$ has the RadonNikodym property [RNP] if and only for every positive finite 
measure space $(T, \Sigma, \mu)$ and any $m: \Sigma \rightarrow E, m$ a countably additive vector measure with locally bounded average range such that $m \ll \mu$, there is an integrable $f: T \rightarrow E$ such that $m(S)=\int_{S} f d \mu, S \in \Sigma$.

In what follows we give several characterizations of QSMC spaces possessing the RNP. The next theorem was proved for Banach spaces by Davis and Phelps [5] and Huff [9].

THEOREM. If $E$ is a QSMC space, the following are equivalent:

(1) $E$ has the RNP.

(2) Every bounded set in $E$ is dentable.

(3) Every bounded closed convex set in $E$ is dentable.

Proof.

$(2) \Rightarrow(1)$ : Follows from the RNT.

$(3) \Leftrightarrow(2)$ : This follows from the extension of a result of Reiffel [18] to QSMC spaces, if $K \subset E$ and $\bar{c}(K)$ is dentable, then $K$ is dentable.

$(1) \Rightarrow(2)$ : Assume $C^{\prime}$ is a nondentable bounded set in $E$, then $C \equiv \bar{c}\left(C^{\prime}\right)$ is nondentable. Let $K$ be the disked hull, $d(C)$, of $C$ and take $\hat{K} \supset K$ by the QSMC property. Then $C$ is not dentable in $E_{\widehat{K}}$, thus, as in Huff [9], there exists a positive finite measure space $(T, \Sigma, \mu)$ and a countably additive vector measure $m: \Sigma \rightarrow E_{\widehat{K}}$ with finite total variation such that $A_{T}(m) \subset C$ and such that $m$ has no density in $E_{\widehat{K}}$. Then $m: \Sigma \rightarrow E$ is a countably additive vector measure and by assumption has a density in $E$. Thus we can assume $m$ has locally relatively compact average range in $C \subset E$ and hence in $E_{K}$, but this contradicts the RNT for Banach spaces.

Next we give an extension of the strongly exposed point characterization of Banach spaces with the RNP given in [17] and show that for some more general spaces the result must be modified slightly.

Definition 7. If $C \subset E$, then $\left(E^{*}\right)_{C}$ is the collection of linear functionals on $E$ whose restrictions to $C$ are continuous.

Definition 8. If $C \subset E$, then $x \in C$ is an $\left(E^{*}\right)_{c}$-strongly exposed point of $C$ if there is an $x^{*} \in\left(E^{*}\right)_{C}$ such that $x^{*}(x)>x^{*}(C \backslash\{x\})$ and $x^{*}\left(x_{\alpha}\right) \rightarrow x^{*}(x)$, where $\left\{x_{\alpha}\right\}$ is a net in $C$, implies $x_{\alpha} \rightarrow x$ in $E$.

The next result was obtained by Saab [20] for Fréchet spaces where the integral involved was a Bochner type integral. Our proof is accomplished for QSMC spaces by modifying only slightly the proofs of lemmas given by Phelps [17], so the proofs of the lemmas are omitted. We will state the lemmas as the statements must be 
altered slightly as well. We also point out that the first three lemmas hold for general quasicomplete locally convex topological vector spaces and the SMC property is mainly used thereafter to insure we can take a sequence of nested slices which intersect in a point.

THEOREM. E has the RNP $\Leftrightarrow$ Every closed bounded convex set, $C$, is the closed convex hull of its $\left(E^{*}\right)_{C}$ strongly exposed points.

Definition 9. If $K \subset E, x^{\prime} \in E^{\prime}, \alpha>0$ then define $S\left(x^{\prime}, \alpha, K\right)=$ $\left\{x \in K: x^{\prime}(x) \geqq \sup _{y \in K} x^{\prime}(y)-\alpha\right\}$. Such a set is called a slice of $K$.

Lemma. A subset $K$ of $E$ is dentable if and only if for every $\rho \in\{\rho\}$ and $\varepsilon>0$, there is a slice $S\left(x^{\prime}, \alpha, K\right)$ with $x^{\prime} \in E, \alpha>0$ such that the $\rho$-diam of $S\left(x^{\prime}, \alpha, K\right)$, denoted $\delta_{\rho}\left(S\left(x^{\prime}, \alpha, K\right)\right)$, is less than $\varepsilon\left(\delta_{\rho}\left(S\left(x^{\prime}, \alpha, K\right)\right)<\varepsilon\right)$.

Lemma. If $T: E \rightarrow E$ is an isomorphism of $E$ and $S\left(x^{\prime}, \alpha, C\right)$ is a slice of $C$ such that for some $\rho \in\{\rho\}$ and $\varepsilon>0$, we have $\delta_{\rho}\left(S\left(x^{\prime}\right.\right.$, $\alpha, C))<\varepsilon$, then $T\left(S\left(x^{\prime}, \alpha, C\right)\right)$ is a slice of $T(C)$ and for $x \in S\left(x^{\prime}, \alpha\right.$, C) we have $T\left(S\left(x^{\prime}, \alpha, C\right)\right) \subset T\left(S_{\varepsilon}^{\rho}(x)\right)$.

Lemma. Suppose every closed bounded set in $E$ is dentable, $C$ is closed bounded convex, $x^{\prime} \in E^{\prime}$ and $C \backslash N_{x^{\prime}} \neq \varnothing$, where $N_{x^{\prime}}=$ $\left\{x: x^{\prime}(x)=0\right\}$. Then there is a $y^{\prime} \in E^{\prime}$ and $\beta>0$ such that $\delta_{\rho}\left(S\left(y^{\prime}\right.\right.$, $\beta, C))<\varepsilon$ and $S\left(y^{\prime}, \beta, C\right) \cap N_{x^{\prime}}=\varnothing$.

Lemma. Suppose every closed bounded set in $E$ is dentable and $C$ is closed bounded and convex. Then $C$ is the closed convex hull of its $E^{\prime}$-denting points.

A point $x \in C$ is a $E^{\prime}$-denting point of $C$ if for each $\varepsilon>0$ and $\rho \in\{\rho\}$ there is a slice $S\left(x^{\prime}, \alpha, C\right)$ of $\rho$-diameter less than $\varepsilon$ which contains $x$.

Lemma. Suppose every closed bounded set in $E$ is dentable and $S\left(x^{\prime}, \alpha, C\right)$ is a slice of the closed bounded convex set $C,\left\|x^{\prime}\right\|_{K}=$ $1\left(K=2 d(C),\left\|x^{\prime}\right\|_{K}=\sup _{k \in K}\left|x^{\prime}(k)\right|\right)$. Let $1>\varepsilon>0, \rho \in\{\rho\}$ and $\delta>0$, then there is a slice $S\left(y^{\prime}, \beta, C\right)$ such that $\delta_{\rho}\left(S\left(y^{\prime}, \beta, C\right)\right)<\delta$, $\| x^{\prime}-$ $y^{\prime} \|_{K}<\varepsilon$ and $\left\|x^{\prime}\right\|_{K}=\left\|y^{\prime}\right\|_{K}=1, S\left(y^{\prime}, \beta, C\right) \subset S\left(x^{\prime}, \alpha, C\right)$.

REMARK. Here we take $C_{1}=\bar{c}\left(S\left(x^{\prime}, \alpha, C\right) \cup\left[\lambda K \cap N_{x^{\prime}}\right]\right), \lambda>2 / \varepsilon$, and proceed as in Phelps [17]. We use $K$ instead of the unit ball in the Banach space case. 
Lemma. Let $C$ be a closed bounded convex set in $E$. Suppose for every slice $S\left(x^{\prime}, \alpha, C\right), \rho \in\{\rho\}, \delta>0$ and $\varepsilon>0$, there is a slice $S\left(y^{\prime}, \beta, C\right)$ such that $\delta_{\rho}\left(S\left(y^{\prime}, \beta, C\right)\right)<\delta, S\left(y^{\prime}, \beta, C\right) \subset S\left(x^{\prime}, \alpha, C\right)$ and $\left\|x^{\prime}-y^{\prime}\right\|_{K}<\varepsilon$ where $K=2 d(C)$. Then every slice $S\left(x^{\prime}, \alpha, C\right)$ of $C$ contains an $\left(E^{*}\right)_{K}$ strongly exposed point.

REMARK. (1) The above lemmas show that the RNP implies that every closed bounded convex set $C$ is the closed convex hull of its $\left(E^{*}\right)_{C}$-strongly exposed points. The other direction is trivial since $\left(E^{*}\right)_{C}$-strongly exposed points are denting points.

(2) The functionals giving the slices in the proof of the last lemma are only converging to some function in sup norm on $K$. This function will be linear in $K$ and we can extend it to a function which is continuous on $K$ and linear on $E$, i.e.; in $\left(E^{*}\right)_{K}$.

(3) Even in Banach spaces there are linear functionals which are continuous on a closed bounded convex set and which are not continuous on the disked hull of the set. Hence our result is actually stronger than stated above since the functionals we obtain are continuous on the disked hull of the sets. In fact they can be chosen to be continuous on any preassigned boundeds et.

EXAMPLE. Let $\left\{e_{n}\right\}$ be the standard unit vector basis for $c_{0}$ and define $\delta_{n}=\sum_{i=1}^{n} e_{i}$. Let $K=\bar{c}\left(\left\{\delta_{n}\right\}_{n=1}^{\infty}\right) \subset c_{0}$, then we have $K=\left\{\left\{\alpha_{i}\right\}_{1}^{\infty} \epsilon\right.$ $C_{0}: \alpha_{1}=1$ and $\left.\alpha_{i} \geqq \alpha_{i+1}\right\}$. Now define $f\left(\left\{\alpha_{\imath}\right\}_{1}^{\infty}\right)=\sum_{i=1}^{\infty}(-1)^{i} \alpha_{i}$ for all $\left\{\alpha_{i}\right\} \in K$ and extend linearly to $c_{0}$. Then $f$ is continuous on $K$.

Claim. $f$ is not continuous on $d(K)$.

Proof. Let $x_{n}=\sum_{k=1}^{n}\left((-1)^{k} / 2 n\right) \delta_{k}=(\underbrace{0,1 / 2 n, 0,1 / 2 n, 0, \cdots, 1 / 2 n}_{2 n}$, $0,0, \cdots)$ then $x_{n} \in d(K)$ and $x_{n} \rightarrow 0$ yet $f\left(x_{n}\right)=1 / 2 n-0+1 / 2 n-0+$ $\cdots+1 / 2 n=1 / 2$.

We now show that we cannot necessarily expect continuity of the linear functionals on the whole space.

Theorem. Let $\left\{B_{i}\right\}$ be sequence of Banach spaces and $E=\mathbf{X}_{i} B_{i}$, then $E$ has the RNP if and only if $B_{i}$ has the RNP for all $i$.

Proof. $(\Rightarrow)$ If there is a $B_{i}$ which doesn't have the RNP, then take $K_{i} \subset B_{i}$ closed bounded convex and not dentable in $B_{i}$. Then define $K=K_{i} \times \times_{j \neq i}\{0\}$ and it is easily verified that $K$ is not dentable in $E$.

$(\Longleftrightarrow)$ If $(T, \Sigma, \mu)$ is a countably additive vector measure with locally bounded average range, then $\pi_{i} \circ m: \Sigma \rightarrow B_{i}$ is a countably 
additive vector measure in $B_{i} . \pi_{i} \circ m$ has locally compact average range by the RNP and RNT in $B_{i}$. Take a set $S \in \Sigma^{+}$and construct a sequence $\left\{S_{i}\right\} \subset \Sigma^{+}, S_{i+1} \subset S_{i} \subset S$, for all $i$ such that $A_{S_{i}}\left(\pi_{i}(m)\right)$ is relatively compact in $B_{i}$ and such that $S^{\prime}=\bigcap_{i} S_{i} \in \Sigma^{+}$. This can be done as in Maynard [14, Page 457]. Then we have $A_{S^{\prime}}(m)$ is relatively compact in $E$. Hence apply RNT for QSMC spaces to obtain density for $m$.

Remark. Peck [16, Page 275] showed that if $\left\{B_{i}\right\}_{i=1}^{\infty}$ is a collection of nonreflexive Banach spaces and $E=X_{i} B_{i}$, then there is a $C \subset E$ closed bounded convex with no $E^{\prime}$-support points. A point $x \in C$ is an $E^{\prime}$-support point of $C$ if there is an $x^{\prime} \in E^{\prime}$ such that $x^{\prime}(x)=\sup _{c \in C} x^{\prime}(c)$. Thus if $B_{i}=l^{1}$ for all $i$, we see that $E$ has the RNP; thus there is a closed bounded convex set which is the closed convex hull of its $\left(E^{*}\right)_{C}$-strongly exposed points and yet has no $E^{\prime}$ support points.

Corollary. Assume $F$ is a Fréchet space, let $F_{n}$ be the completion of $F / \operatorname{ker} \rho_{n}$, and assume $F_{n}$ has the RNP for all $n$, then $F$ has the RNP.

Corollary. Assume $E$ is QSMC space. If for each bounded disk $B$, there is a $\hat{B} \supset B$ bounded closed disked set such that $E_{\widehat{B}}$ has the RNP, then $E$ has the RNP.

These corollaries follow immediately from the above theorems.

II. In this section we extend a few results of Bishop and Phelps [1] and Davis, Figiel, Johnson, Pelczynski [4] to QSMC spaces. Some analogous results along these lines for Fréchet spaces are found in Peck [16]. The proofs here are straightforward consequences of the corresponding results in Banach spaces.

TheOREM. Assume $E$ is a QSMC space, $C$ is a closed convex set in $E$, and $C_{1}$ is any other closed bounded convex set containing $C$. Then the $\left(E^{*}\right)_{C_{1}}$-support points are dense in the boundary of $C$. (Here $x \in C$ is an $\left(E^{*}\right)_{C_{1}}$-support point of $C$ if there is an $x^{*} \in\left(E^{*}\right)_{C_{1}}$ such that $x^{*}(x)=\sup _{c \in C} x^{*}(C)$. We also point out that if $E$ is not a normed space then the support points are dense in the whole set $C$ and since the Banach space theorem is already known we assume in the following proof that $E$ is not a normed space.)

Proof. Take any $z \in C, \rho \in\{\rho\}$ and $\delta>0$, then we want $z_{1} \in S_{\delta}^{\rho}(z) \cap C$ and $x^{*} \in\left(E^{*}\right)_{C_{1}}$ such that $x^{*}\left(z_{1}\right)=\sup x^{*}(C)$. Take 
$\omega \in S_{i}^{\rho}(z) \backslash C$, let $K=d\left(C_{1} \cup\{\omega\}\right)$ and take $\hat{K} \supset K$ by the QSMC property. Now there is some $u$ in $C$ on the line joining $z$ and $\omega$ which is an $E_{\widehat{K}}$ boundary point of $C$. Take $\varepsilon>0$ such that for all $x, y \in K$ such that $x-y \in S_{\varepsilon}^{\widehat{K}}(0)$ implies $x-y \in S_{\delta / 2}^{o}(0)$. Then by [1, Page 29] there exists a $z_{1}$ in the boundary of $C$ in $E_{\widehat{K}}$ intersect the $S_{\varepsilon}^{\widehat{K}}(u)$ and an $x^{\prime} \in\left(E_{\widehat{K}}\right)$ such that $x^{\prime}\left(z_{1}\right)=\sup x^{\prime}(C)$. Now $z_{1}$ is contained in $C \cap S_{\delta}^{o}(z)$ and $x^{\prime}$ can be extended linearly to all of $E$, say to $x^{*}$. Then $x^{*} \in\left(E^{*}\right)_{C_{1}}$ and $x^{*}\left(z_{1}\right)=\sup x^{*}(C)$.

TheOREM. Suppose $C_{1}, C_{2} \subset E, C_{1}$ closed bounded convex, $C_{2}$ is bounded $D=d\left(C_{1} \cup C_{2}\right)$, and $D_{1}$ is any closed bounded disked set containing $D$. If there is an $x^{*} \in\left(E^{*}\right)_{D_{1}}$ such that $\sup x^{*}\left(C_{1}\right)<$ inf $x^{*}\left(C_{2}\right)$, then for every $\varepsilon>0$, there is a $y^{*} \in\left(E^{*}\right)_{D_{1}}$ and $y \in C_{1}$ such that $y^{*}(y)=\sup y^{*}\left(C_{1}\right)<\inf y^{*}\left(C_{2}\right)$ and the $\sup _{x \in D_{1}}\left|\left\langle x^{*}-y^{*}, x\right\rangle\right|<\varepsilon$.

Proof. Choose real numbers $a, b, c, d$ such that $\sup x^{*}\left(C_{1}\right)<$ $a<b<c<d=\inf x^{*}\left(C_{2}\right)$ and take $\varepsilon<\inf [b-a / 2, d-c / 2]$, then by Horvath [8, Page 248] there is an $x^{\prime} \in E^{\prime}$ such that $\sup _{x \in D_{1}} \mid\left\langle x^{*}-x^{\prime}\right.$, $x\rangle \mid<\varepsilon / 6$, hence $\sup x^{\prime}\left(C_{1}\right)<b<c<\inf x^{\prime}\left(C_{2}\right)$. Take $\hat{D} \supset D_{1}$ by the QSMC property. Since $x^{\prime} \in E^{\prime}$, it is also in $\left(E_{\widehat{D}}\right)^{\prime}$; thus by Bishop and Phelps [1, Page 30] there is a $y^{\prime} \in\left(E_{\widehat{D}}\right)^{\prime}$ and $y \in C_{1}$ such that $y^{\prime}(y)=\sup y^{\prime}\left(C_{1}\right)<\inf y^{\prime}\left(C_{2}\right)$ and $\sup _{x \in D_{1}}\left|\left\langle y^{\prime}-x^{\prime}, x\right\rangle\right|<\varepsilon / 3$. We now have $\left|\left\langle y^{\prime}-x^{*}, \quad x\right\rangle\right| \leqq\left|\left\langle y^{\prime}-x^{\prime}, \quad x\right\rangle\right|+\left|\left\langle x^{*}-x^{\prime}, \quad x\right\rangle\right|\langle\varepsilon / 2$, for all $x \in D_{1}$. Now extend $y^{\prime}$ to be linear on $E$, call the extension $y^{*}$. Then $y^{*} \in\left(E^{*}\right)_{D_{1}} y^{*}(y)=\sup y^{*}\left(C_{1}\right)<\inf y^{*}\left(C_{2}\right)$ and $\sup _{x \in D_{1}} \mid\left\langle y^{*}-x^{*}\right.$, $x>\mid<\varepsilon$.

THEOREM. If $K \subset E$ is a closed bounded disked set and $K_{1} \supset K$ $i s$ any closed bounded disked set, then for every $x^{*} \in\left(E^{*}\right)_{K_{1}}$ and $\varepsilon>0$, there exists a $y^{*} \in\left(E^{*}\right)_{K_{1}}$ such that $y^{*}$ is a support functional of $K$ and $\sup _{x \in K_{1}}\left|\left\langle x^{*}-y^{*}, x\right\rangle\right| \leqq \varepsilon$.

Proof. Take $x^{*} \in\left(E^{*}\right)_{K_{1}}$ and $\hat{K} \supset K_{1}$ by QSMC property, then $x^{*} \in\left(E_{\widehat{K}}^{*}\right)_{K_{1}}$ (i.e.: $x^{*}$ is linear on $E_{\widehat{K}}$ and continuous on $K_{1}$ since the topologies of $E$ and $E_{\widehat{K}}$ agree on $K_{1}$ ). Now $K_{1}$ is symmetric, hence by Horvath [8, Page 248] there exists a $z^{\prime} \in\left(E_{\widehat{K}}\right)^{\prime}$ such that $\sup _{x \in K_{1}}\left|\left\langle x^{*}-z^{\prime}, x\right\rangle\right|<\varepsilon / 2$. Therefore we can apply a result of Bishop and Phelps [1, Page 31] to obtain a $y^{\prime} \in\left(E_{\widehat{K}}\right)^{\prime}$ and a $y \in K$ such that $y^{\prime}(y)=\sup y^{\prime}(K)$ and $\sup _{x \in K_{1}}\left|\left\langle z^{\prime}-y^{\prime}, \quad x\right\rangle\right|<\varepsilon / 2$. Now extend $y^{\prime}$ to $y^{*} \in\left(E^{*}\right)_{K_{1}}$ and we have that $\sup _{x \in K_{1}}\left|\left\langle x^{*}-y^{*}, x\right\rangle\right|<$ $\varepsilon / 2+\varepsilon / 2=\varepsilon$ and $y^{*}$ is a support functional for $K$.

The next two results are extensions of results from [4] and require some additional notation and a technical result from their paper which we state without proof. Assume $E$ is a QSMC space 
and $\mathrm{W} \subset E$ is a convex, symmetric bounded set. Let $K=d(W)$ and take $\hat{K} \supset K$ by the QSMC property. For every $n$, define $U_{n}=2^{n} W+2^{-n} \hat{K}$. Then $U_{n}$ defines an equivalent norm on $E_{\widehat{K}}$, call the norm $\|\cdot\|_{n}$. For each $x \in E_{\widehat{K}}$ define $\|x\|=\left(\sum_{1}^{\infty}\|x\|_{n}^{2}\right)^{1 / 2}$. Let $Y=\left\{x \in E_{\widehat{K}}:\|x\|<\infty\right\}$ and denote by $C$ the set of $x \in Y$ such that $\|x\| \leqq 1$ and by $j: Y \rightarrow E_{\widehat{K}}$ the identity embedding.

LEMMA. (1) $W \subseteq C$.

(2) $(Y,\|\cdot\|)$ is a Banach space and $j$ is continuous.

(3) $j^{\prime \prime}\left(Y^{\prime \prime}\right) \rightarrow E_{\widehat{K}}^{\prime \prime}$ is $1-1$ and $\left(j^{\prime \prime}\right)^{-1}\left(E_{\widehat{K}}\right)=Y$.

(4) $Y$ is reflexive if and only if $W$ is relatively weakly compact. The proof is found in [4].

THEOREM. If $L$ is a locally convex topological vector space, $E$ is a QSMC space; $T: L \rightarrow E$ is a weakly compact operator, then there exists a reflexive Banach space $R$ and continuous linear operators $T_{1}$ and $T_{2}$ such that $T=T_{2} \circ T_{1}, T_{1}: L \rightarrow R, T_{2}: R \rightarrow E$.

Proof. Take $U$ an absolutely convex balanced neighborhood of 0 in $L$ such that $T(U)$ is relatively weakly compact in $E$. Let $K=\overline{T(U)}$ and take $\hat{K} \supset K$ by the QSMC property. Denote by $i$ the continuous embedding of $E_{\widehat{K}}$ into $E$. Now $i^{-1} \circ T: L \rightarrow E_{\widehat{K}}$ is a continuous linear transformation since a net convergent to zero in $L$ is eventually in $U$, and the topologies of $E$ and $E_{\widehat{K}}$ agree on $K$. Thus the map $i^{-1} \circ T: L \rightarrow E_{\widehat{K}}$ is a weakly compact operator into a Banach space, which implies by [4, Page 314] that there exists a reflexive Banach space $R$ and operator $F_{1}, F_{2}$ such that $i^{-1} \circ T=F_{2} \circ F_{1}$ where $F_{1}: L \rightarrow R, F_{2}: R \rightarrow E_{\widehat{K}}$. Then let $T_{1}=F_{1}, T_{2}=i \circ F_{2}$ and we have the appropriate factorization.

THEOREM. If $K \subset E$ is a weakly compact set, then $K$ is affinely homeomorphic to a subset of a reflexive Banach space.

Proof. Let $K_{1}=d(K)$ and take $\hat{K}_{1} \supset K_{1}$ by the QSMC property. Then by the lemma, $Y$ is reflexive. Therefore $K^{\prime}=j^{-1}\left(K_{1}\right)$ is weakly compact, $\left.j\right|_{K^{\prime}}$ is the homeomorphism for $K$ between $E_{\widehat{K}_{1}}$ and $Y$ and we need only compose this map with $i^{-1}$ of the preceding proof.

Remarks. It follows from Grothendieck [6, Ex. 2, p. 61] that weakly compact subsets of QSMC spaces are Eberlein compacts (i.e., a compact hausdorff space which is homeomorphic to a weakly compact subset of a Banach space); thus one can obtain results along the lines of Lindenstrauss [12] such as: If $K \subset E$ is a weakly 
compact subset of a QSMC space then $K$ is affinely homeomorphic to a weakly compact subset of a $c_{0}(\Gamma)$ space for some $\Gamma$.

In closing we shall discuss some examples and nonexamples of QSMC spaces and the RNP.

(1) An obvious collection of spaces one would hope to be QSMC spaces, in light of a result mentioned earlier, are not. Take any separable infinite dimensional Banach space $X$ and consider $E=\left(X^{\prime}, \sigma\left(X^{\prime}, X\right)\right)$. Then $E$ has a fundamental sequence of bounded sets and the closed bounded sets are metrizable and complete. Thus if $E$ has the MC property Grothendieck's result would say that $E$ has the QSMC property. To see that $E$ is not a QSMC space, let $U_{X}$, be the closed unit ball in $X^{\prime}$. Then $U_{X^{\prime}}$ is closed bounded (in fact compact) in $E$. If $K$ is a closed bounded disked set which contains $U_{X^{\prime}}$, then there is a $\lambda>0$ such that $U_{X^{\prime}} \subset K \subset \lambda U_{X^{\prime}}$ and hence $E_{K}$ is isomorphic to $X^{\prime}$. But $U_{X^{\prime}}$ is compact in $E$ hence the topologies of $E_{K}$ and $E$ cannot agree on $U_{X^{\prime}}$.

It is an easy matter to define our integral and RNP for quasicomplete locally convex spaces with bounded sets metrizable. In which case it follows immediately that dual spaces of separable Banach spaces with the weak-* topology have the RNP (since bounded sets are relatively compact).

(2) If $X$ is a separable Banach space, consider $E=B_{S}(X)$, the space of bounded operators on $X$ with the strong operator topology. $E$ is a quasicomplete locally convex space with a fundamental sequence of bounded sets and bounded sets are metrizable. Since subspaces of spaces with the SMC property must have the SMC property, we shall show that $E$ has a closed subspace without the SMC property. Take $x_{1} \in X,\left\|x_{1}\right\|=1$ and for every $x^{\prime} \in X^{\prime}$ define $T_{x^{\prime}}: X \rightarrow X$ by $T_{x^{\prime}}(x)=x^{\prime}(x) x_{1}$. Let $S=\left\{T_{x^{\prime}}: x^{\prime} \in X^{\prime}\right\}$, then $S$ is a closed subspace of $E$ (in fact $S$ is a closed subspace of the compact operators on $X$ with the strong operator topology). By the argument given in (1) above we see that $S$ doesn't have the SMC property.

(3) One can see immediately from the definition [7, Page 176] that if $E$ is a quasi-barreled locally convex topological vector space, then $E$ is quasi-normable if and only if the strong dual of $E$ is a SMC space. We note that if $E$ is barreled then the strong dual is not necessary.

(4) A complete $(D F)$ space is bornological if and only if it is an $(L F)$ space [7, Page 172]. Now $(D F)$ spaces have a fundamental sequence of bounded sets and hence bornological $(D F)$ spaces are SMC spaces.

(5) As indicated earlier arbitrary topological direct sums of 
spaces with the SMC property have the SMC property. Hence it is easy to see that a topological direct sum of a collection of Banach spaces with the RNP has the RNP.

(6) Many spaces of distributions and other inductive limit spaces are SMC spaces and are also Montel spaces. These spaces by the RNT all possess the RNP.

\section{REFERENCES}

1. E. Bishop and R. R. Phelps, The Support Functionals of Convex Set; Proceedings Symposium Pure Math American Mathematical Society, Vol. 7 (Convexity) (1963), pp. $27-35$.

2. G. Chi, A geometric characterization of Fréchet spaces with the RNT; Proc. Amer Math. Soc., 48 (1975), 371-380.

3. G. Chi, RNT for Fréchet spaces, (preprint).

4. W.J. Davis, T. Figiel, W.B. Johnson and A. Pelczynski, Factoring weakly compact operators, J. Functional Analysis, 17 No. 3, (1974) 311-327.

5. W. J. Davis and R. R. Phelps; The Radon-Nikodym property and dentable sets in Banach spaces, Proc. Amer. Math. Soc., 45 (1974), 119-122.

6. A Grothendieck, Sur les espaces $(F)$ et $(D F)$, Summa Brasiliensis, Mathematical, 3, fas 6, (1954), 57-122.

7. — Topological Vector Spaces; Gordon and Breach, London U. K., 1973.

8. J. Horvath, Topological Vector Spaces and Distributions, Vol. 1, Addison-Wesley Publishing Co., Reading, Massachusetts, 1966.

9. R. Huff, Dentability and the Radon-Nikodym property, Duke Math., 41 (1975), 111-114.

10. R. Huff and P. Morris, Geometric characterization of the RNP in Banach spaces, Studia Math. (to appear).

11. Bengt Josepson, Weak sequential convergence in the dual of a Banach space does not imply norm convergence, Bull. Amer. Math. Soc. 81, No. 1 (1975), 166-168.

12. J. Lindenstrauss, Weakly compact sets-their topological properties and the Banach spaces they generate, Annals Math. Studies 69, Princeton University Press, (1972), 235-273. 13. H. Maynard, A geometric characterization of Banach spaces with the RadonNikodym property, Trans. Amer. Math. Soc., 185 (1973), 493-500.

14. A Radon-Nikodym theorem for operators valued measures, Trans. Amer. Math. Soc., 173 (1972), 449-463.

15. S. Moedemo and J. J. Uhl, Jr., Radon-Nikodym theorems for the Bochner and Pettis integrals, Pacific J. Math. 38 (1971), 531-536.

16. N. T. Peck, Support points in locally convex spaces, Duke Math. J., 38 (1971), 271-278.

17. R. R. Phelps, Dentability and extreme points in Banach spaces, J. Functional Analysis, 16 (1974), 78-90.

18. M. Rieffel, Dentable Subsets of Banach Spaces with Applications to a RNT, Functional Analysis (B. R. Gelbaum, Editor), Thompson Publishing Company, (1971), Washington.

19. The Radon-Nikodym theorem for the Bochner integral, Trans. Amer. Math. Soc., 131 (1968), 466-487.

20. E. Saab, Points Extrémaux et Propriété de Radon-Nikodym dans les espaces de Fréchet Dentables, Séminare choquet, $13^{\circ}$ annee 1973-74 n ${ }^{\circ} 19,14 \mathrm{p}$.

Received December 15, 1975.

THE University of UtAH. 


\section{PACIFIC JOURNAL OF MATHEMATICS}

\section{EDITORS}

RICHARD ARENS (Managing Editor)

University of California

Los Angeles, California 90024

\section{R. A. Beaumont}

University of Washington

Seattle, Washington 98105
J. DugundjI

Department of Mathematics

University of Southern California

Los Angeles, California 90007

D. Gilbarg and J. Milgram

Stanford University

Stanford, California 94305

\section{ASSOCIATE EDITORS}
E. F. BECKENBACH
B. H. NeumanN
F. WOLF
K. YosHIDA

\section{SUPPORTING INSTITUTIONS}

\author{
UNIVERSITY OF BRITISH COLUMBIA \\ CALIFORNIA INSTITUTE OF TECHNOLOGY \\ UNIVERSITY OF CALIFORNIA \\ MONTANA STATE UNIVERSITY \\ UNIVERSITY OF NEVADA \\ NEW MEXICO STATE UNIVERSITY \\ OREGON STATE UNIVERSITY \\ UNIVERSITY OF OREGON \\ OSAKA UNIVERSITY
}

\author{
UNIVERSITY OF SOUTHERN CALIFORNIA \\ STANFORD UNIVERSITY \\ UNIVERSITY OF HAWAII \\ UNIVERSITY OF TOKYO \\ UNIVERSITY OF UTAH \\ WASHINGTON STATE UNIVERSITY \\ UNIVERSITY OF WASHINGTON \\ AMERICAN MATHEMATICAL SOCIETY
}

The Supporting Institutions listed above contribute to the cost of publication of this Journal, but they are not owners or publishers and have no responsibility for its content or policies.

Mathematical papers intended for publication in the Pacific Journal of Mathematics should be in typed form or offset-reproduced, (not dittoed), double spaced with large margins. Please do not use built up fractions in the text of your manuscript. You may however, use them in the displayed equations. Underline Greek letters in red, German in green, and script in blue. The first paragraph or two must be capable of being used separately as a synopsis of the entire paper. Items of the bibliography should not be cited there unless absolutely necessary, in which case they must be identified by author and Journal, rather than by item number. Manuscripts, in triplicate, may be sent to any one of the editors. Please classify according to the scheme of Math. Reviews, Index to Vol. 39. All other communications should be addressed to the managing editor, or Elaine Barth, University of California, Los Angeles, California, 90024.

The Pacific Journal of Mathematics expects the author's institution to pay page charges, and reserves the right to delay publication for nonpayment of charges in case of financial emergency.

100 reprints are provided free for each article, only if page charges have been substantially paid. Additional copies may be obtained at cost in multiples of 50 .

The Pacific Journal of Mathematics is issued monthly as of January 1966. Regular subscription rate: $\$ 72.00$ a year (6 Vols., 12 issues). Special rate: $\$ 36.00$ a year to individual members of supporting institutions.

Subscriptions, orders for back numbers, and changes of address should be sent to Pacific Journal of Mathematics, 103 Highland Boulevard, Berkeley, California, 94708.

PUBLISHED BY PACIFIC JOURNAL OF MATHEMATICS, A NON-PROFIT CORPORATION

Printed at Kokusai Bunken Insatsusha (International Academic Printing Co., Ltd.), 8-8, 3-chome, Takadanobaba, Shinjuku-ku, Tokyo 160, Japan. 


\section{Pacific Journal of Mathematics \\ Vol. 65, No. $2 \quad$ October, 1976}

Andrew Adler, Weak homomorphisms and invariants: an example .......... 293

Howard Anton and William J. Pervin, Separation axioms and metric-like

functions ............................................. 299

Ron C. Blei, Sidon partitions and p-Sidon sets .................... 307

T. J. Cheatham and J. R. Smith, Regular and semisimple modules ........... 315

Charles Edward Cleaver, Packing spheres in Orlicz spaces .............. 325

Le Baron O. Ferguson and Michael D. Rusk, Korovkin sets for an operator on a

space of continuous functions ............................. 337

Rudolf Fritsch, An approximation theorem for maps into Kan fibrations ....... 347

David Sexton Gilliam, Geometry and the Radon-Nikodym theorem in strict

Mackey convergence spaces .................................

William Hery, Maximal ideals in algebras of topological algebra valued

functions ...................................... 365

Alan Hopenwasser, The radical of a reflexive operator algebra ........... 375

Bruno Kramm, A characterization of Riemann algebras................. 393

Peter K. F. Kuhfittig, Fixed points of locally contractive and nonexpansive

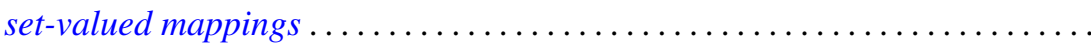

Stephen Allan McGrath, On almost everywhere convergence of Abel means of

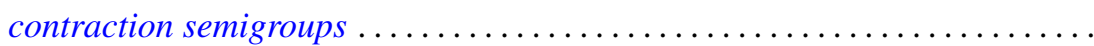

Edward Peter Merkes and Marion Wetzel, A geometric characterization of

indeterminate moment sequences............................ 409

John C. Morgan, II, The absolute Baire property ................... 421

Eli Aaron Passow and John A. Roulier, Negative theorems on generalized convex approximation .................................... 437

Louis Jackson Ratliff, Jr., A theorem on prime divisors of zero and characterizations of unmixed local domains ..............

Ellen Elizabeth Reed, A class of $T_{1}$-compactifications................... 471

Maxwell Alexander Rosenlicht, On Liouville's theory of elementary

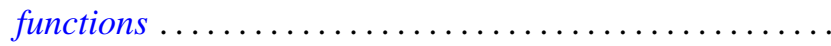

Arthur Argyle Sagle, Power-associative algebras and Riemannian

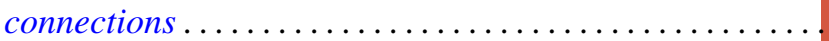

Chester Cornelius Seabury, On extending regular holomorphic maps from Stein manifolds...

Elias Sai Wan Shiu, Commutators and numerical ranges of powers of operators ...................................

Donald Mark Topkis, The structure of sublattices of the product of $n$ lattices ... 525

John Bason Wagoner, Delooping the continuous $K$-theory of a valuation

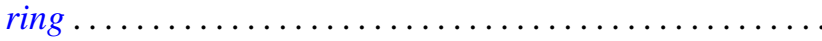

Ronson Joseph Warne, Standard regular semigroups...........

Anthony William Wickstead, The centraliser of $E \otimes_{\lambda} F \ldots$. 